Andressa Silva ${ }^{\mathrm{a}}$

Lucimare Ferraz $^{\mathrm{a}}$

Sinval Adalberto Rodrigues-Junior ${ }^{\mathrm{a}}$

a Universidade Comunitária da Região de Chapecó. Chapecó, SC, Brasil.

Contato:

Sinval Adalberto Rodrigues-Junior

E-mail:

rodriguesjunior.sa@unochapeco.edu.br

O trabalho integra a dissertação intitulada "Saúde do Trabalhador na Atenção Básica: ações e educação permanente", defendida em julho de 2015 na Universidade Comunitária da Região de Chapecó (Unochapecó).

O trabalho não foi subvencionado nem apresentado em reunião científica.

Os autores declaram não haver conflitos de interesses.

\section{Ações em Saúde do Trabalhador desenvolvidas na Atenção Primária no município de Chapecó, Santa Catarina}

\author{
Worker's health actions developed by \\ Primary Health Care in Chapecó, Santa Catarina, Brazil
}

\section{Resumo}

Objetivo: conhecer as ações de Saúde do Trabalhador desenvolvidas nos centros de saúde da Atenção Primária do município de Chapecó (SC) e verificar se elas estão incorporadas às rotinas dos serviços de saúde da Atenção Primária. Métodos: estudo descritivo com enfoque qualitativo, com utilização de entrevista semiestruturada e técnica de análise de conteúdo. Foram entrevistados dez sujeitos, com funções de coordenadores e de recepcionistas, de quatro Centros de Saúde urbanos e um rural, em 2014. Resultados: os entrevistados revelaram que, entre as diversas ações em Saúde do Trabalhador preconizadas pelo Ministério da Saúde, apenas as notificações de agravos relacionados ao trabalho (acidentes de trabalho e doenças ocupacionais) são realizadas. Conclusões: Mediante agendamento, as rotinas de trabalho na Atenção Primária estão centradas em atendimentos ao usuário e as ações desenvolvidas nos centros de saúde contemplam apenas os Programas de Saúde básicos. Entre as dificuldades enfrentadas para a realização de ações em Saúde do Trabalhador, o pouco conhecimento a respeito do tema se converte em um forte argumento para justificar a própria ausência dessas ações.

Palavras-chave: saúde do trabalhador; sistema único de saúde; atenção primária à saúde.

\begin{abstract}
Objective: to be acquainted with the Worker's Health actions carried out at Primary Care Health Centers in Chapecó, Santa Catarina, Brazil, and to verify if they are incorporated into the routines of the Primary Care Health services. Methods: descriptive qualitative study using semi-structured interview and content analysis. Ten individuals, coordinators and receptionists, from urban and rural Health Centers, were interviewed in 2014. Results: the interviewees reported that, among the Worker's Health actions advocated by the Ministry of Health, the centers only carried out the notifications on work-related injuries (work accidents and occupational diseases). Conclusions: the Primary Care Health Centers' routines are focused on attending to users, and contemplate only basic Health Programs. The scarce knowledge on occupational health becomes a strong argument to explain the very lack of Workers' Health actions.
\end{abstract} Keywords: workers health; occupational health; unified health system; primary health care. 


\section{Introdução}

O cuidado à saúde dos trabalhadores, sob a ótica da esfera pública, emergiu em meio à transição democrática da década de 1980, almejando constituir um novo campo de atuação e intervenção da rede pública de serviços de saúde no Brasil. Por meio da instituição de novas legislações, objetivou-se mudar o complexo quadro de saúde da população ${ }^{1,2}$.

A instituição do Sistema Único de Saúde (SUS), concebido pela Constituição Federal de 1988 e regulamentado pela Lei Orgânica da Saúde em 1990, reforçou o conceito ampliado de saúde, incorporando ao novo sistema questões de saúde do trabalhador. Também foi atribuída ao SUS a responsabilidade de coordenar as ações de prevenção, promoção e assistência à saúde no país ${ }^{3-5}$.

A criação da Norma Operacional Básica de Saúde do Trabalhador gerou o arcabouço legal para tratar das questões pertinentes à saúde do trabalhador no SUS, orientando e instrumentalizando as ações e serviços de saúde para esse propósito 6 . Em 2002, diante da necessidade de melhor organizar e institucionalizar as referidas ações nos três níveis de gestão do SUS, criou-se a Rede Nacional de Atenção Integral à Saúde do Trabalhador (Renast) ${ }^{7}$.

As ações de Saúde do Trabalhador (ST) presente na rede do SUS objetiva a promoção e a proteção da saúde do trabalhador, por meio de ações de vigilância dos riscos existentes nos ambientes e condições de trabalho, dos agravos à saúde e da organização e prestação da assistência aos trabalhadores. Ela compreende procedimentos de diagnóstico, tratamento e reabilitação de forma integrada na rede de serviços do SUS, e seu objeto de estudo e intervenção são as relações entre o trabalho e a saúde ${ }^{8}$.

Contudo, a existência de leis não tem sido suficiente para transformar a realidade da ST, em especial, no contexto do SUS. A inserção da ST na esfera pública tem se configurado um desafio permanente para o desenvolvimento técnico-operacional das práticas de saúde. Esse desafio pode ser observado em todos os níveis de atenção como, por exemplo, na assistência, na vigilância à saúde, no tratamento das informações, na gestão dos serviços, na condução de políticas locais e programáticas, e na formação e educação permanente dos profissionais da saúde ${ }^{9,10}$.

Vasconcellos e Machado ${ }^{9}$ mencionam a marginalização da ST no SUS ao longo dos anos. Ainda, afirmam que esse campo da saúde pública não superou a fragmentação estrutural da rede de atenção à ST, e sequer foi concebido como uma política de saúde inovadora. Diante dessas fragilidades e com o intuito de fortalecê-lo, em 2012, o Ministério da
Saúde instituiu a Política Nacional de Saúde do Trabalhador e da Trabalhadora (PNSTT), definindo os princípios, as diretrizes e as estratégias a serem observados pelas três esferas da gestão dos serviços de saúde pública ${ }^{11}$.

A PNSTT ${ }^{11}$, enquanto instrumento norteador, propõe a estruturação da Renast no contexto da Rede de Atenção à Saúde (RAS). Ela pressupõe que ações de ST sejam desenvolvidas junto à atenção primária em saúde, em serviços de urgência e emergência e na atenção especializada (ambulatorial e hospitalar).

A Atenção Primária à Saúde (APS) no SUS revela-se reorientadora do modelo de atenção à saúde no Brasil em função de seu modelo de atenção e pelo constante incremento de estratégias, mostrando potencialidades para o desenvolvimento de ações de ST no SUS ${ }^{10,12}$.

Os serviços da APS, caracterizados como a principal porta de entrada dos usuários no SUS, são essenciais para o desenvolvimento de ações em ST articuladas com a rede de atenção à saúde, desde que haja o reconhecimento do usuário como trabalhador e a compreensão do trabalho enquanto determinante do processo saúde-doença ${ }^{13}$.

Os problemas de saúde relacionados ao trabalho podem ser detectados primeiramente na APS, já que seus serviços estão presentes no território em que os trabalhadores residem e, eventualmente, trabalham. Além disso, de acordo com a Portaria $\mathrm{n}^{0} 1.823$, que institui a PNSTT ${ }^{11}$, os serviços de APS são primordiais na garantia da integralidade na atenção à saúde do trabalhador, mediante ações de proteção, com articulação e construção conjunta de protocolos, linhas de cuidado e matriciamento na assistência da saúde do trabalhador.

Assim, este estudo objetivou conhecer as ações em saúde do trabalhador desenvolvidas nos Centros de Saúde da Atenção Primária do município de Chapecó, em Santa Catarina.

\section{Métodos}

Trata-se de uma pesquisa descritiva com enfoque qualitativo, cujo cenário de estudo envolveu os centros de saúde (CS) da Rede de Atenção Primária do município de Chapecó. Essa rede é composta por 26 CS, 20 urbanos e 6 rurais, que comportam 41 Equipes de Saúde da Família (ESF), e encontra-se classificada e distribuída geograficamente nas regiões norte, sul, leste e oeste da cidade. O estudo buscou contemplar todos os territórios de saúde do município, sendo realizado em cinco CS, contemplando um por região (norte, sul, leste e oeste) e um CS rural. 
Como critério de seleção dos CS participantes do estudo, utilizou-se a maior abrangência populacional, de acordo com levantamento prévio realizado no Sistema de Informação da Atenção Básica (Siab). Ainda, a fim de contemplar a realidade rural, elencou-se um centro de saúde rural, independentemente de seu território, porém, seguindo o critério da maior abrangência populacional.

Foram sujeitos da pesquisa os coordenadores e os profissionais da recepção dos CS, que mantêm o primeiro contato com os usuários, totalizando dez sujeitos. Os coordenadores foram incluídos por conhecerem os processos e fluxos de trabalho dos serviços e, assim, poderem responder de forma satisfatória aos questionamentos da pesquisa. Os trabalhadores de saúde que fazem a recepção do usuário (geralmente um técnico de enfermagem) têm um papel de suma importância no acolhimento e encaminhamento das demandas dos trabalhadores que adentram nos CS. Por isso, esses profissionais também foram incluídos neste estudo.

O procedimento adotado para a coleta de dados foi a entrevista semiestruturada, conforme roteiro pré-estabelecido, que questionava: i) a rotina de trabalho e de assistência prestada no CS; ii) as ações voltadas à saúde do trabalhador que a equipe do CS desenvolve; iii) existência de dificuldades (e quais) para a implementação de ações de Saúde do Trabalhador pela equipe do CS iv) as demandas trazidas pelos usuários para o CS. As entrevistas ocorreram nos CS selecionados, de forma individual, após serem agendadas com os sujeitos da pesquisa.

No início de cada entrevista, o estudo foi brevemente apresentado aos sujeitos antes de solicitar a eles a assinatura, em duas vias, do termo de consentimento livre e esclarecido e do termo para uso de imagens e áudio. Todas as entrevistas foram gravadas e transcritas logo após seu término. Os depoimentos de cada sujeito foram identificados por letras $(\mathrm{C}$ - coordenadores dos CS; $\mathrm{R}$ - profissionais que atuam na recepção dos CS), seguidos do número ordinal correspondente a sua realização. Os dados foram coletados entre setembro e novembro de 2014.

Utilizou-se como técnica a Análise de Conteúdo proposta por Minayo ${ }^{14}$. Sendo assim, os dados coletados foram submetidos à análise seguindo três etapas: i) pré-análise: os dados foram organizados por meio de leituras sistematizadas dos relatos das entrevistas e foram determinadas as unidades de análise, partindo das questões do roteiro das entrevistas; ii) exploração do material: consistiu na classificação das unidades de análise a fim de alcançar o núcleo de compreensão do texto; a partir de então, distribuíram-se trechos do texto de forma a contemplar as categorias, já pré-definidas pelo roteiro de perguntas dos questionários; iii) interpretação dos resultados: nesse momento os pesquisadores dialogaram com a fundamentação teórica adotada, estabelecendo correlações com as informações provenientes de outros estudos.

Esta pesquisa foi aprovada pelo Comitê de Ética em Pesquisa da Unochapecó, sob o parecer $\mathrm{n}^{\mathrm{O}}$ 155/2014 e autorizada pela Secretaria de Saúde de Chapecó.

\section{Resultados}

\section{Rotinas dos Centros de Saúde}

As rotinas nos serviços de saúde da APS estão centradas em atendimentos ao usuário, mediante agendamento. As ações desenvolvidas nos CS contemplam os principais programas de saúde (Saúde da Criança, Saúde da Mulher, Saúde do Homem, Saúde do Idoso, entre outros) preconizados pelo Ministério da Saúde (MS), como mostram os depoimentos a seguir:

Aqui é uma unidade de Atenção Básica [Primária], então a gente trabalha com consultas agendadas. Tem os programas de hipertensos. A gente acompanha hipertensos, diabéticos, saúde da criança, saúde da mulher. (C1)

Tudo agendado, algumas urgências a gente encaixa porque os pacientes faltam. (C4)

Nós somos responsáveis pelos cadastros de novos pacientes, pacientes transferidos de outras unidades pra nossa, cartão nacional do SUS, atualização dos cadastros também, agendamento de consultas, atendimento telefônico, agendamento de dentista. O primeiro contato com o paciente quando ele chega na unidade pra encaminhar pra cada setor também é de nossa responsabilidade. (R3)

Bom, desempenho várias funções: atendimento ao público geral, desde crianças, adolescentes, jovens, adultos, idosos, agendamento de consultas, retorno de exames também, cuido das agendas dos médicos, informações, tudo que for necessário na questão de saúde. (R4)

\section{Ações de ST desenvolvidas nos CS}

Os entrevistados relataram as ações de ST realizadas nos serviços de saúde da APS, entre elas, a praticada é a notificação de agravos relacionados ao trabalho (acidentes de trabalho e doenças ocupacionais), como se observa nas falas abaixo:

O que a gente tá fazendo são as notificações. Eu tô sempre frisando. Reunião passada eu disse "precisa notificar". Paciente chega ali pra fazer um curativo, pergunta se se acidentou no trabalho, se foi em casa, onde foi. (C2) 
Na realidade a gente tá engatinhando ainda, mal as notificações a gente consegue realizar. A gente tem um profissional que tem especialidade em saúde do trabalhador, mas nem as notificações a pessoa não faz, então na realidade a gente tem ainda essa dificuldade de conseguir agregar isso ao trabalho. (C3)

As ações [de ST] basicamente são as notificações. [...] quando são identificadas - isso é um problema relacionado ao trabalho - são notificadas. (C5)

Quando identificamos o acidente de trabalho, já chamamos as colegas. Elas já vêm com a ficha e já registram que foi um acidente de trabalho. (R2)

\section{Dificuldades para o desenvolvimento de ações em ST}

Pôde-se verificar, conforme trechos das entrevistas listados a seguir, que a falta de conhecimento a respeito da temática ST é impedimento ao desenvolvimento das ações, ao mesmo tempo que acaba sendo forte argumento para justificar a ausência do desenvolvimento de ações em ST pelos CS.

Eu não sei te informar se tem algum método diferenciado que a gente tenha que usar aqui na frente [recepção dos CS]! Mas, a princípio, se ele [usuário] não se identificar como trabalhador, pra nós aqui são todos pacientes! (R2)

Não sei se a equipe está totalmente preparada, vai muito do investigar, de perguntar, eu acho que a gente faz pouco disso - de perguntar, saber por que ele tá com aquele problema. (R3)

Eu vejo que a dificuldade começa no balcão, quando a gente faz o acolhimento, ninguém pergunta onde foi que se machucou. "Foi um acidente de trabalho?". É falta de capacitação mesmo dos funcionários pra fazer isso. (C4)

Eu acredito que, quem sabe, o ensino teórico mesmo sobre o que são acidentes de trabalho, por exemplo, muitas pneumoconioses relacionadas ao trabalho, nem o profissional médico que tá atendendo o paciente ali há anos identifica que esse problema é relacionado a sua atividade laboral. Então eu acho que às vezes é relacionado à falta de estudo, à orientação. (C5)

\section{Demandas dos usuários}

As queixas e/ou demandas trazidas pelos usuários aos serviços de saúde da APS não são relacionadas às atividades laborais que desenvolvem, portanto, não são reconhecidas pelos servidores enquanto problemas de ST. Como se pode observar a seguir, os depoimentos dos entrevistados não apontam queixas e/ou agravos relacionados ao trabalho.

A gente acompanha hipertensos, diabéticos, saúde da criança, saúde da mulher. (C1)

Lombalgia, muita gestante, tabagismo intenso, obesidade, problemas conjugais, depressão. Os maiores problemas são hipertensão, diabetes e problema mental. (C5)
Eles chegam e dizem que é porque dói aqui, dói lá, dói um monte de coisa [...] eles vêm no posto e somatizam sintomas pra que a gente consiga uma consulta pra eles, e na verdade não é nada disso. (R2)

Muito pouco dos nossos pacientes vem pra prevenção, a maioria vem já com o problema especifico. Muitos pacientes com problemas comuns. A gente tem bastante da ortopedia, pressão, diabetes, não tomam a medicação da forma correta, se alimentam da forma errada, a maioria dos nossos pacientes vem com esses sintomas. (R3)

\section{Discussão}

Os depoimentos apontam para o desenvolvimento de vários programas de saúde específicos na APS (Saúde da Criança, Saúde da Mulher, Saúde do Homem, Saúde do Idoso, entre outros). Esses programas são preconizados pela Norma Operacional da Assistência à Saúde (NOAS-SUS), de modo a atender às demandas e às necessidades de saúde da população brasileira ${ }^{12,15}$.

No entanto, os entrevistados não explicitam o desenvolvimento de ações baseadas nas necessidades locais de saúde da população atendida nos serviços da APS, o que diverge do propósito do SUS, que tem a APS como a principal porta de entrada dos usuários. Cabe à APS o papel de organizar e articular o cuidado na RAS, de modo a garantir que a população tenha acesso a uma atenção à saúde de qualidade, próxima de onde os usuários moram, trabalham, estudam e vivem ${ }^{12}$.

Cabe ressaltar, ainda, que, com importância similar à dos programas supracitados e desenvolvidos na APS, a Renast, instituída em 2002, incorporou o desenvolvimento de ações de prevenção, promoção, vigilância, assistência e reabilitação em saúde do trabalhador, em todos os níveis de atenção do SUS ${ }^{7}$. Porém, nos depoimentos não foi possível identificar a prática dessas ações nas rotinas de trabalho da APS.

A deficiência no desenvolvimento de ações em ST tem sido apontada como um desafio para o SUS. Embora seja possível evidenciar alguns avanços, a implementação e/ou a consolidação das ações em saúde do trabalhador na APS ainda demanda esforços, uma vez que se almeja a garantia da atenção integral à saúde dos trabalhadores de forma resolutiva e coordenada com os demais níveis de complexidade $^{13}$.

Devido à capilaridade da rede de serviços do SUS preconizada e regulamentada, os serviços da APS deveriam abranger o trabalhador brasileiro de maneira mais próxima aos territórios onde ele mora e trabalha. Contudo, estudos sugerem que a APS ainda 
não incorporou a ST em seu processo de trabalho, de modo a atender às necessidades de saúde que emergem do contexto de vida e trabalho da população ${ }^{16,17}$.

Outros autores afirmam que, apesar de estar regulamentada desde 1990 e contar com arcabouço jurídico relativamente completo, a ST no SUS ainda se encontra marginalizada ${ }^{9}$. A falta de mudanças efetivas na práxis devida à política de Estado instituída tem feito que as legislações acerca do tema venham sendo apenas atualizadas, reafirmando atribuições já vigentes, e que o campo da ST no SUS não seja consolidado $^{18}$. A PNSTT, instituída em 2012, reafirma o compromisso da integralidade na atenção à saúde do trabalhador, pressupondo a inserção de ações de ST em todas as instâncias e pontos da RAS do SUS, incluindo a $\mathrm{APS}^{11}$.

Dentre as ações de ST junto à APS, a PNSTT prevê o reconhecimento e mapeamento das atividades produtivas no território; o reconhecimento e a identificação da população trabalhadora; o perfil sócio-ocupacional no território e riscos e impactos (perfil de morbimortalidade) potenciais à saúde dos trabalhadores; a identificação da rede de apoio social aos trabalhadores no território; a identificação e registro da situação de trabalho; a identificação e registro da ocupação e do ramo de atividade econômica dos usuários das unidades e serviços de atenção primária em saúde; a identificação da relação entre o trabalho e o problema de saúde apresentado pelo usuário; a notificação dos agravos relacionados ao trabalho no Sistema de Informação de Agravos de notificação (Sinan) e no Sistema de Informação da Atenção Básica (Siab) e emissão da Comunicação de Acidente do Trabalho (CAT); a articulação com as equipes técnicas e os Centros de Referência em Saúde do Trabalhador (Cerest); a definição e a implantação de condutas e manejo assistenciais (de promoção e de vigilância em ST); e a incorporação de conteúdos de ST nas estratégias de capacitação e de educação permanente para as equipes da atenção primária em saúde ${ }^{11}$.

Neste estudo, observou-se que a notificação de agravos relacionados ao trabalho (acidentes de trabalho e doenças ocupacionais) emergiu como uma atividade desenvolvida pelos profissionais, ficando evidente que as ações preconizadas pela PNSTT estão sendo desenvolvidas parcialmente. Contudo, quando indagados sobre suas rotinas de trabalho, os entrevistados não mencionaram a realização do procedimento notificatório de agravos relacionados ao trabalho como uma ação em ST.

A ST, enquanto campo de atuação e intervenção da rede pública de serviços de saúde no Brasil, tem por objetivo mudar o complexo quadro de saúde da população trabalhadora ${ }^{1,2}$. Porém, observa-se que, mesmo diante de muitas legislações instituídas no SUS, a implantação das ações, políticas e programas de forma plena e efetiva não tem ocorrido ${ }^{19,20}$.

Quanto ao procedimento de "notificação", o SUS vem lançando esforços desde 2004 para a melhoria das informações em ST com a implementação da Portaria $n^{0} 777^{21}$. Essa portaria estabeleceu os procedimentos técnicos para a notificação compulsória de agravos à saúde do trabalhador no Sinan na rede de serviços do SUS, definindo os seguintes agravos: acidente de trabalho grave (incluindo o acidente de trabalho fatal e com crianças e adolescentes); acidente de trabalho com exposição a material biológico; intoxicação exógena (incluindo intoxicações por agrotóxicos, chumbo e benzeno); dermatose ocupacional; lesão por esforço repetitivo (LER)/doença osteomuscular relacionada ao trabalho (Dort); perda auditiva induzida por ruído (Pair); transtorno mental relacionado ao trabalho; pneumoconioses; e câncer ocupacional.

A sistematização das informações do Sinan permite o diagnóstico dos acidentes de trabalho e das doenças ocupacionais, subsidiando as equipes de saúde na identificação dos riscos aos quais os trabalhadores estão expostos e, por consequência, na tomada de decisões para intervir na ocorrência desses agravos ${ }^{22}$.

Ainda com vistas à melhoria da qualidade dos dados e ampliação dos registros, em 2014 o MS atualizou a lista do Sinan que contempla os agravos e/ ou eventos de saúde pública passíveis de notificação em todo país, incluindo os agravos relacionados ao trabalho ${ }^{23,24}$. Embora avanços nos sistemas de informação em saúde no Brasil sejam identificados, pesquisas assinalam que os acidentes e doenças relacionadas ao trabalho são subnotificados, gerando a necessidade de melhoria, tanto na cobertura dos registros como na qualidade das informações lançadas no $\operatorname{Sinan}{ }^{25,26}$.

Evangelista et al. ${ }^{16}$ também identificaram que a notificação de agravos à saúde no Sinan representa um importante avanço na construção do perfil epidemiológico dos trabalhadores brasileiros, apesar de ainda haver subnotificação das doenças ocupacionais e dos acidentes de trabalho. Vale ressaltar que o registro de agravos relacionados ao trabalho é realizado pela APS de modo burocrático, a fim de atender às exigências legais, sem considerar as contribuições de caráter epidemiológico que os dados em ST oferecem para o subsídio das ações de intervenção ${ }^{27}$. Segundo Santana e Ferrite (2013), a capacidade limitada de reconhecimento da relação causal ocupacional com os problemas de saúde dos usuários é uma das causas de sub-registro e de subnotificação. Além disso, o fato de a notificação compulsória ser 
realizada apenas após a ocorrência do acidente de trabalho ou doença ocupacional deixa evidente a lacuna no desenvolvimento das ações de prevenção e promoção da saúde do trabalhador ${ }^{28}$.

Quanto às possíveis dificuldades que a equipe de saúde apresenta para a realização de ações em ST, além das limitações dos profissionais da saúde sobre a temática ST, os depoimentos deixam evidente que, muitas vezes, o usuário é atendido nos serviços de saúde, mas não é reconhecido como um trabalhador. O reconhecimento do usuário enquanto trabalhador é imprescindível para a atenção integral à saúde desse público. No acolhimento, nas consultas, nas visitas domiciliares e demais atividades em saúde, é essencial que os profissionais da saúde conheçam a atividade laboral e obtenham uma breve história profissional dos usuários, possibilitando, assim, a identificação de riscos a sua saúde e estabelecendo possíveis relações entre a doença ou a queixa apresentada e o subsídio para a definição de ações de intervenção ${ }^{29}$.

Lacaz $^{30}$ indica, dentre as fragilidades impostas pela implantação tardia de uma política de saúde efetiva, dificuldades estruturais para organizar os serviços de atenção aos trabalhadores, como a formação de recursos humanos e aquisição de materiais. Nesse sentido, a deficiência na formação em nível de graduação e na educação permanente em saúde para atuar em ST aparece com muita ênfase. Autores reforçam a necessidade de revisão dos projetos pedagógicos dos cursos de graduação em saúde para a consolidação da ST no SUS e desafiam ainda a gestão do SUS e seus trabalhadores para efetivar a Educação Permanente em Saúde ${ }^{16}$.

Outrossim, Fernandes ${ }^{31}$ constatou que os currículos dos cursos de enfermagem contemplam os conteúdos essenciais à formação do enfermeiro no que diz respeito à ST. Entretanto, os documentos curriculares analisados diferem do discurso dos sujeitos entrevistados, deixando evidente a necessidade de aproximar as ementas da proposta original dos currículos. Chiavegatto ${ }^{32}$ reforça que a falta de atenção à coleta de dados referentes ao histórico ocupacional dos usuários gera uma lacuna nas práticas cotidianas, em especial de médicos e enfermeiros.

Marques e colaboradores ${ }^{33}$ também mencionam que o processo de formação nas instituições de ensino, tanto em nível de graduação como de pós-graduação ainda é incipiente quando se trata de ST e sua relação com a APS. Segundo eles, essa situação pode ser observada na produção acadêmica, ainda restrita, quando comparada a outras linhas de estudo. Visto que os enfoques dados estão mais voltados à Medicina do Trabalho e à Saúde Ocupacional, alguns autores têm sugerido a revisão dos currículos de graduação, visando a contemplar a temática em suas diversas dimensões, inclusive na saúde pública.

Além dos aspectos voltados à formação profissional, o reconhecimento dos trabalhadores na APS pode ser facilitado por meio da utilização de instrumentos de territorialização. Estudos relatam que a territorialização mostra-se como um importante instrumento para a identificação do usuário trabalhador na APS. Ela possibilita o reconhecimento e/ou identificação dos problemas de saúde provenientes dos processos produtivos e condições de trabalho, possibilitando o desenvolvimento de ações de vigilância e assistência adequadas às necessidades de saúde da população. Além de possibilitar o mapeamento do local e o cadastramento das famílias, a territorialização permite às equipes de saúde amplo conhecimento acerca de determinado território, subsidiando a organização do processo de trabalho e o planejamento das ações de saúde da APS 5,19,34.

De igual importância, o acolhimento realizado nos serviços de saúde é essencial para a humanização e a integralidade da assistência. Quando realizado visando a identificar as necessidades e condições de vida dos usuários, ele pode fornecer subsídios às equipes de saúde na identificação das queixas e/ou agravos em saúde apresentados pelos usuários quando possuírem relação com suas atividades laborais ${ }^{5,19,35}$. Assim, o desafio desse campo da saúde transcende as abordagens assistenciais, necessitando de abordagens interdisciplinares, intersetoriais e acadêmicas que fortaleçam o sistema de saúde e produzam mudanças eficazes para a promoção da saúde dos trabalhadores ${ }^{1,5,36}$.

Por fim, quando questionados sobre as demandas e/ou queixas que os usuários traziam até o CS, os entrevistados não apontaram a demanda de queixas e/ou agravos relacionados ao trabalho. De acordo com Nehmy e Dias ${ }^{37}$, as equipes de saúde apresentam dificuldades em relacionar as queixas e/ou agravos à atividade laboral. Esses aspectos requerem do SUS maior cautela para garantir atenção integral à saúde do trabalhador. Nesse sentido, a identificação do perfil dos trabalhadores e do processo saúde-doença impõe desafios ao setor público de saúde para responder a essas dificuldades ${ }^{37}$.

Outros estudos também mostram que as mudanças no "mundo do trabalho" vêm refletindo no perfil de adoecimento da população trabalhadora, ocasionando demandas de saúde novas, diversificadas e desafiadoras ao Estado. Esses estudos mencionam a urgência em se incorporar práticas de atenção à ST na Atenção Primária e ressaltam a necessidade de infraestrutura nos serviços, bem como a ampliação e a qualificação dos profissionais que atuam na $\mathrm{APS}^{5,10}$. 
Percebe-se que muitas questões que envolvem as relações trabalho-saúde-doença-ambiente e os impactos que os processos produtivos acarretam aos territórios de atuação das equipes da $\mathrm{AB}$ geram demandas de saúde que já vêm sendo atendidas pelas equipes da ESF. Portanto, é fundamental que os profissionais da saúde vinculados à ESF ofereçam algum tipo de solução/encaminhamento para essas demandas $^{29}$.

\section{Considerações finais}

As rotinas de trabalho na APS estão centradas em atendimentos ao usuário, mediante agendamento; as ações desenvolvidas nos CS contemplam os principais programas de saúde, contudo não alcançam a ST.

Esse campo, enquanto política pública de saúde, revela-se relativamente novo e, por esse motivo, as ações ainda são muitas vezes desconhecidas pelos profissionais da saúde. Os relatos dos entrevistados revelaram que, dentre as diversas ações em ST preconizadas pelo MS, apenas as notificações de agravos relacionados ao trabalho (acidentes de trabalho e doenças ocupacionais) estão sendo desenvolvidas.

Dentre as dificuldades explicitadas para a realização de ações em ST, a qualificação inadequada dos profissionais da saúde e, por consequência, o conhecimento deficiente acerca da temática são grandes impedimentos, ao mesmo tempo que acaba sendo o forte argumento para justificar a ausência de desenvolvimento dessas ações.

Dessa forma, compreende-se que visualizar o trabalho como fator determinante do processo saúde-doença dos indivíduos e da coletividade e reconhecer o usuário como trabalhador revelam-se essenciais no "fazer diário" da APS. A invisibilidade desses fatores nos serviços públicos de saúde, em especial na Atenção Básica - principal porta de entrada da RAS do SUS - contribui para que a população trabalhadora permaneça desassistida e marginalizada.

Acredita-se que resgatar o papel da Rede de Atenção à Saúde do Trabalhador seria um dos caminhos possíveis para fortalecer a atenção integral aos usuários trabalhadores, bem como, fomentar e potencializar o desenvolvimento das ações em saúde do trabalhador na APS.

De igual importância, a territorialização também se revela como um instrumento importante para sensibilizar os profissionais de saúde da APS quanto ao processo de adoecimento dos usuários trabalhadores. À medida que os processos de trabalho são mapeados e reconhecidos pelos profissionais como potenciais geradores de riscos à saúde dos trabalhadores, eles poderão ser mais bem monitorados pelas equipes de saúde.

\section{Contribuições dos autores}

Os autores contribuíram igualmente na concepção e delineamento do estudo, análise e interpretação dos dados, redação e revisão crítica do conteúdo intelectual do manuscrito.

\section{Referências}

1. Gomez CM. Campo da saúde do trabalhador: trajetória, configurações e transformações. In: Gomez CM, organizador. Saúde do trabalhador na sociedade brasileira contemporânea. Rio de Janeiro: Fiocruz; 2011. p. 23-34.

2. Santos APL, Lacaz FAC. Saúde do Trabalhador no SUS: contexto, estratégias e desafios. In: Gomez, CM, organizador. Saúde do trabalhador na sociedade brasileira contemporânea. Rio de Janeiro: Fiocruz; 2011. p. 87-106.

3. Brasil. Constituição (1988). Constituição da República Federativa do Brasil: promulgada em 5 de outubro de 1988. São Paulo: Saraiva; 1988.

4. Brasil. Lei $\mathrm{n}^{0}$ 8.080, de 19 setembro de 1990. Dispõe sobre as condições para a promoção, proteção e recuperação da saúde, a organização e o funcionamento dos serviços correspondentes e dá outras providências. Diário Oficial da União, Brasília, DF; 1990 [citado em 2013 set. 8]. Disponível em: <https://goo.gl/c2onDh>.

5. Dias EC, Silva TL. Possibilidades e desafios para a atenção integral à saúde dos trabalhadores na Atenção Primária. In: Dias EC, organizador. Saúde do trabalhador na Atenção Primária à Saúde: possibilidades, desafios e perspectivas. Belo Horizonte: Coopmed, 2013a. p. 21-42.

6. Brasil. Portaria $n^{\circ} 3.908$, de 30 de outubro de 1998 Norma Operacional em Saúde do Trabalhador do SUS. Brasília, DF: Ministério da Saúde; 1998.

7. Brasil. Portaria $\mathrm{n}^{0} 1.679$, de 19 de setembro de 2002. Dispõe sobre a ampliação e o fortalecimento da Rede Nacional de Atenção Integral à Saúde 
do Trabalhador no SUS e dá outras providências. Brasília, DF: Ministério da Saúde; 2002.

8. Santa Catarina. Secretaria Estadual da Saúde. Plano estadual de saúde 2012-2015. Florianópolis; 2012.

9. Vasconcellos LCF, Machado JMH. Política Nacional de Saúde do Trabalhador: ampliação do objeto em direção a uma política de Estado. In: Gomez CM, organizador. Saúde do trabalhador na sociedade brasileira contemporânea. Rio de Janeiro: Fiocruz; 2011. p. 37-66.

10. Dias MDA. Compreender o trabalho na Atenção Primária à Saúde para desenvolver ações em Saúde do Trabalhador: o caso de um município de médio porte. Rev bras saúde ocup.2013;38(127):69-80.

11. Brasil. Portaria $n^{0} 1.823$, de 23 de agosto de 2012 . Institui a política nacional de saúde do trabalhador e da trabalhadora. Brasília, DF: Ministério da Saúde; 2012a.

12. Brasil. Ministério da Saúde. Secretaria de Atenção à Saúde. Política Nacional de Atenção Básica. Brasília, 2012b.

13. Dias EC, Silva TL, Chiavegatto CV, Reis JC, Campos AS. Desenvolvimento de ações de saúde do trabalhador no SUS: a estratégia da Rede Nacional de Atenção Integral à Saúde do Trabalhador (Renast). In: Gomez CM, Machado JMH, Pena PGL, organizadores. Saúde do trabalhador na sociedade brasileira contemporânea. Rio de Janeiro: Fiocruz; 2011. p. 107-122.

14. Minayo MCS. O desafio do conhecimento: pesquisa qualitativa em saúde. São Paulo: Hucitec; 2013.

15. Brasil. Ministério da Saúde. Secretaria de Assistência à Saúde. Regionalização da assistência à saúde: aprofundando a descentralização com equidade no acesso. Brasília, DF: Ministério da Saúde; 2001.

16. Evangelista AIB, Pontes AGV, Silva JV, Saraiva AKM. A saúde do trabalhador na atenção primária à saúde: o olhar do enfermeiro. Rev Rene. 2011;12(Especial):1011-20.

17. Dias EC, Silva TL. Contribuições da Atenção Primária em Saúde do para a implementação da Política Nacional de Saúde e Segurança no Trabalho (PNSST). Rev. bras. Saúde Ocup., São Paulo, v. 38, n. 127, Junho de 2013b.

18. Costa D, Lacaz FAC, Jackson Filho JM, Vilela RAG. Saúde do Trabalhador no SUS: para uma política pública. Rev bras saúde ocup. 2013;38(127):11-30.

19. Dias EC, Rigotto RM, Augusto LGS, Cancio J, Hoefel MGL. Saúde ambiental e saúde do trabalhador na atenção primária à saúde, no SUS: oportunidade e desafios. Ciênc saúde coletiva. 2009;6(14):2061-70.

20. Mendes A. Direito como instrumento de efetivação (ou não) do direito à saúde no Brasil: cenário dos desafios ao direito à saúde universal brasileira. Rev direito sanit. 2013;14(2):113-8.
21. Brasil. Portaria n ${ }^{\circ} 777$, de 28 de abril de 2004. Dispõe sobre os procedimentos técnicos para a notificação compulsória de agravos à saúde do trabalhador em rede de serviços sentinela específica, no Sistema Único de Saúde (SUS). Brasília, DF: Ministério da Saúde; 2004.

22. Daneluz SFC. Reabilitação profissional: um olhar interdisciplinar e interinstitucional na atenção integral à saúde do trabalhador [dissertação]. Itajaí: Universidade do Vale do Itajaí; 2011

23. Brasil. Portaria $n^{0} 1.271$, de 6 de junho de 2014. Define a Lista Nacional de Notificação Compulsória de doenças, agravos e eventos de saúde pública nos serviços de saúde públicos e privados em todo o território nacional, nos termos do anexo, e dá outras providências. Brasília, DF: Ministério da Saúde; 2014a.

24. Brasil. Portaria $\mathrm{n}^{\mathrm{O}} 1.984$, de 12 de setembro de 2014. Define a lista nacional de doenças e agravos de notificação compulsória, na forma do Anexo, a serem monitorados por meio da estratégia de vigilância em unidades sentinelas e suas diretrizes. Brasília, DF: Ministério da Saúde; 2014b.

25. Santana VS, Moura MCP, Soares JFS, Guedes $\mathrm{MH}$. Acidentes de trabalho no Brasil: dados de notificação do Sinan 2007-2008. Salvador: Centro Colaborador Universidade Federal da Bahia, Coordenação Geral da Saúde do Trabalhador; 2009.

26. Galdino A, Santana VS, Ferrite S. Os centros de referência em saúde do trabalhador e a notificação de acidentes de trabalho no Brasil. Cad saúde pública. 2012;28(1):145-59.

27. Balista SRR, Santiago SM, Corrêa Filho HR. A atenção à saúde do trabalhador nas unidades básicas de saúde do SUS: estudo de caso em Campinas, São Paulo. Rev bras saúde ocup. 2011;36(124):212-26.

28. Santana, VS, Ferrite, S. Vigilância epidemiológica em saúde do trabalhador. In: Corrêa MJM, organizador. Vigilância em saúde do trabalhador no Sistema Único de Saúde: teorias e práticas. Belo Horizonte: Coopmed; 2013. p. 99-124.

29. Dias EC, Silva TL, Almeida, MHC. Desafios para a construção cotidiana da Vigilância em Saúde Ambiental e em Saúde do Trabalhador na Atenção Primária à Saúde. Cad saúde coletiva. 2012;20(1):15-24.

30. Lacaz FAC. Política Nacional de Saúde do Trabalhador: desafios e dificuldades. In: Bertani I, organizador. O avesso do trabalho II: trabalho, precarização e saúde do trabalhador. São Paulo: Expressão Popular; 2010, p. 199-230.

31. Fernandes MC. A saúde do trabalhador na formação do enfermeiro em uma instituição pública [dissertação]. Rio de Janeiro: Universidade do Estado do Rio de Janeiro; 2013.

32. Chiavegatto CV. Percepção dos profissionais de nível superior da Atenção Primária quanto ao desenvolvimento de ações de Saúde do Trabalhador no SU em Minas Gerais [dissertação]. 
Belo Horizonte: Universidade Federal de Minas Gerais; 2010.

33. Marques CF, Santos DM, Gonçalves FR, Fernandes MC, Souza NVDO. O ensino de graduação e os conteúdos teórico-práticos da saúde do trabalhador. Rev eletr enf. 2012;14(3):494-503.

34. Monken M, Barcellos C. Vigilância em saúde e território utilizado: possibilidades teóricas e metodológicas. Cad saúde pública. 2005;21(3):898906.

35. Barra SAR, Oliveira, LML. O acolhimento na atenção primária à saúde: dispositivo disparador de mudanças na organização do processo de trabalho? Rev APS. 2012;2(15):126-38.

36. Lourenço EAS. Saúde do trabalhador: de quem é essa responsabilidade? Desafios para o Sistema Único de Saúde. In: Oliveira CAH, Bertani IF. Interdisciplinaridade: integração entre saberes e práticas. Franca: Unesp; 2006.

37. Nehmy RMQ, Dias EC. Os caminhos da saúde do trabalhador: para onde apontam os sinais? Rev Med Minas Gerais 2010;20(2):13-23. 